\title{
Mutual collaboration between Institute of Technology Bandung, Indonesia and Gunma Astronomical Observatory, Japan
}

\author{
Osamu Hashimoto ${ }^{1}$, Hakim L. Malasan ${ }^{2}$, H. Taguchi ${ }^{1}$, K. Kinugasa ${ }^{1}$, \\ B. Dermawan ${ }^{2}$, B. Indradjaja ${ }^{2}$, and Y. Kozai ${ }^{1}$ \\ ${ }^{1}$ Gunma Astronomical Observatory, Takayama, Agatsuma, Gunma 377-0702, Japan, \\ email: osamu@astron.pref.gunma.jp \\ ${ }^{2}$ Department of Astronomy, Institute of Technology Bandung, Indonesia \\ email: hakim@as.itb.ac.id
}

\begin{abstract}
Institute of Technology Bandung (ITB), Indonesia and Gunma Astronomical Observatory $(\mathrm{GAO})$, Japan have been proceeding with several programmes of mutual collaboration in the fields of astronomical research and education since 2002. ITB with Bosscha observatory has a great interest in education of astronomy for public people as well as in the university education and research of their own, and GAO is a public observatory operated by Gunma prefecture local government equipped with a $150-\mathrm{cm}$ reflector and some smaller telescopes, which are capable of scientific research of high grade. We will report some of our cooperative activities including the remote accessing of the telescopes of each observatory by each other, which can provide opportunities for astronomical experiences of the opposite hemisphere for various people of each country. Some scientific collaboration works such as common instruments and data analysis systems developed on both sites are also reported.
\end{abstract}

Keywords. Instrumentation: miscellaneous, instrumentation: spectrographs, astronomy in Indonesia, remote observing, public education in astronomy

\section{Introduction}

Gunma Astronomical Observatory (GAO) was established in Takayama village, which is located about $140 \mathrm{~km}$ northwest of Tokyo ( $\mathrm{N} 36^{\circ} 35^{\prime} 47^{\prime \prime}$, E $138^{\circ} 58^{\prime} 22^{\prime \prime}$ ), by Gunma prefecture local government in 1999. Its main telescope is a 150-cm reflector on an azimuth-elevation mounting, which is one of the most advanced telescopes in Japan with some powerful observation instruments, such as an infrared camera and a high resolution spectrograph (Hashimoto et al. 2002, 2005). GAO is not operated only for scientific researches by professional researchers but also for public education of general people. An eye piece system for public star gazing is equipped even on the $150-\mathrm{cm}$ telescope on the basis of the latter point of view. There are more telescopes prepared for researchers and public visitors; a 65 -cm reflector on an equatorial mounting, six 25-30-cm reflectors, and some 15 -cm refractors.

Institute of Technology Bandung (ITB) is the most leading university in Indonesia in the fields of science and technology. It has the largest department of astronomy in Indonesia as well as an outstanding academic astronomical observatory, Bosscha observatory which was established in 1928. Basically ITB and Bosscha observatory are academic institutions for scientific research and university education of astronomy, but ITB is much interested in the public education as well (Wiramihardja 2003).

There are some common points in the characteristics of ITB and GAO. In fact, both are expected to have similar functions in both scientific research and public education of 


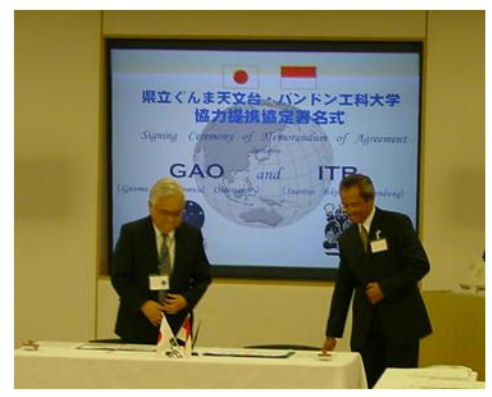

Signing ceremony on 1 July, 2002 at GAO

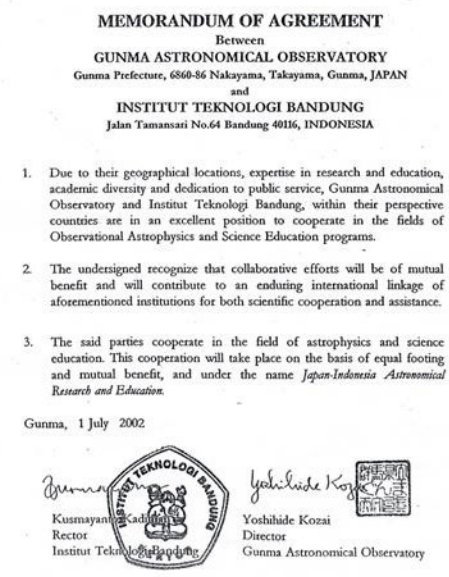

Figure 1. Memorandum of Agreement between ITB and GAO, signed on 1 July 2002.

astronomy. Based on such common circumstances of us, ITB and GAO have kicked off our mutual collaboration programs. Dr Kusmayanto Kadiman, Rector of ITB and Prof. Yoshihide Kozai, Director of GAO have signed the Memorandum of Agreement for the collaboration programs on 1 July 2002 at GAO (Fig. 1).

Since then some concrete programs have been put into practice as our mutual collaboration activities. They are (1) development and operation of a high resolution spectrograph on the GAO 150-cm reflector; (2) development and operation of identical low resolution spectrographs both for Bosscha observatory and GAO telescopes; (3) development and operation of the same reduction and analysis systems both at ITB and GAO for sharing the observational data and the methods with which those data can be handled; (4) mutual collaboration in the studies of astronomy and astrophysics with the use of above mentioned facilities; (5) development and operation of remote telescope system between ITB and GAO, and (6) mutual exchange programmes of staff members of both sides.

\section{Facilities for scientific research}

As a part of scientific programs in our collaboration, a high resolution spectrograph GAOES (Gunma Astronomical Observatory Echelle Spectrograph) is designed and built for the GAO 150-cm telescope (Hashimoto et al. 2005). It provides an optical spectrum of a spectral resolution $(\lambda / \delta \lambda)$ up to 100,000 for wavelengths of $360-1000 \mathrm{~nm}$ on a $4096 \times$ 2048 pixel CCD detector, covering a wavelength range of about $190 \mathrm{~nm}$ by a single exposure (Fig. 2). Such a performance indicates that GAOES can be regarded as one of the most advanced instrument of this type for a 1-2-m class telescope. With the use of this new instrument we have already started some scientific works. For example, studies of binary systems (Puri Jatmiko et al. 2005) and evolved stars in the post-AGB phase (Takeda et al. 2005) are actively going in these years.

Two low resolution spectrographs GCS (Gunma Compact Spectrograph) for the GAO 65 -cm reflector and BCS (Bosscha Compact Spectrograph) for the Bosscha 60-cm refractor or $45 \mathrm{~cm}$ reflector have been built (Fig. 3). Spectral resolution of those spectrographs 

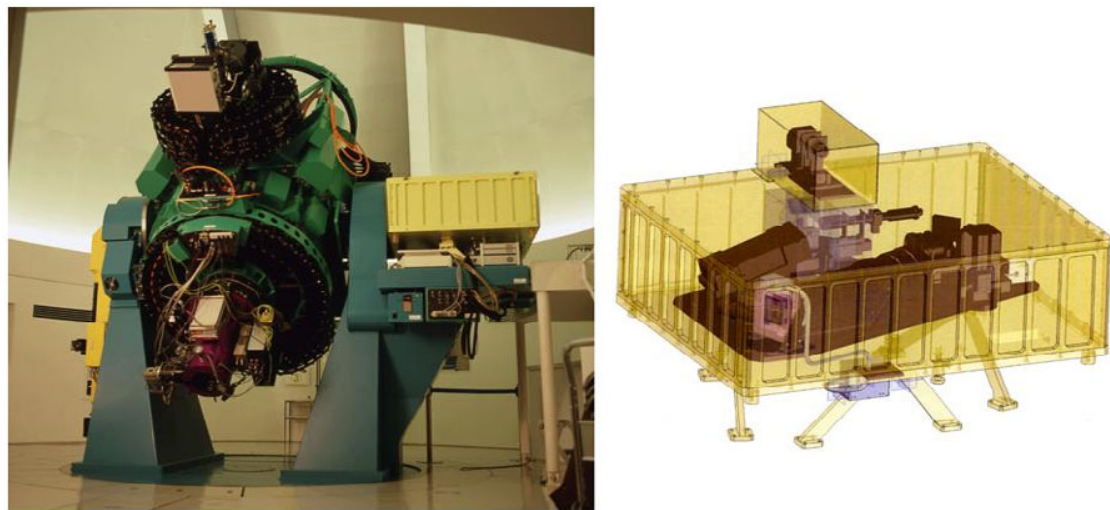

Figure 2. GAOES at Nasmyth focus of the GAO 150-cm telescope (left), and a schematic view of its inside (right).
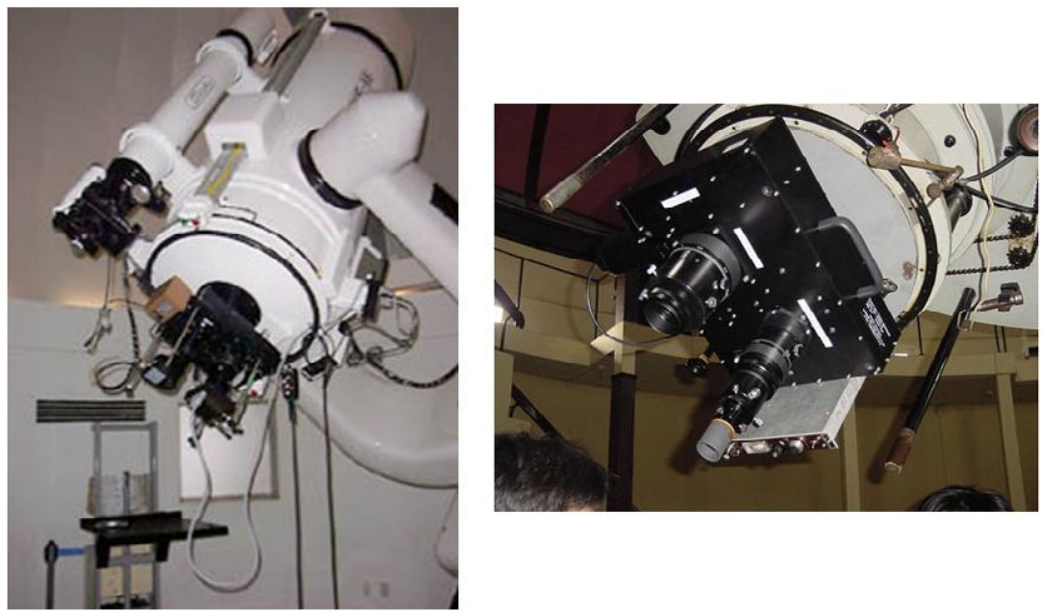

Figure 3. GCS on the GAO 65-cm reflector (left), and BCS on the 60-cm refractor at Bosscha observatory.

is about 500 or 2000 which can be chosen by two different gratings. As those two spectrograph are completely the same, common methods can be developed and applied in the observation and handling of the data at both observatories. We are not intending to use these spectrographs only in various scientific researches, but in some activities for education activities for students and public people.

Also computer systems which are basically the same have been developed both at ITB and GAO for sharing the data of our collaboration studies and for providing the tools for reduction and analysis of those data in the same manner (Kinugasa et al. 2005). We are expecting very effective developments in our cooperative studies with those common facilities.

\section{Remote telescope system and public education}

The location of Bosscha observatory S $6^{\circ} 49^{\prime} 32^{\prime \prime}$, E $107^{\circ} 36^{\prime} 58^{\prime \prime}$ indicates that stars in southern hemisphere that can not be seen in Japan, can be observed at Bosscha observatory, and the north stars which can not be seen in Indonesia vice versa. This point 

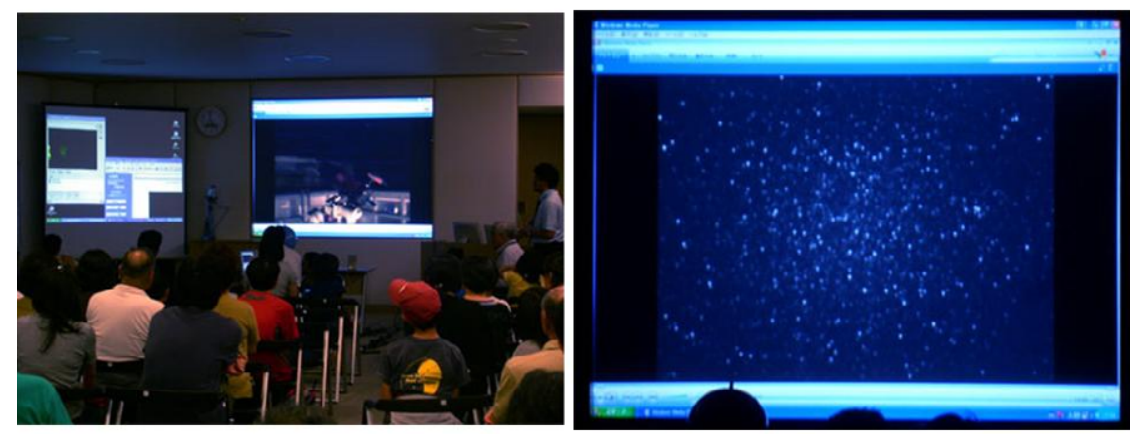

Figure 4. About 100 people gathering at GAO in Japan for the remote star observation of the southern hemisphere from Indonesia (left), and a real time image of $\omega$ Cen projected on the screen at GAO (right), which is sent from a remote control telescope at Bosscha observatory.

is very much useful in our collaborative activities, especially in the public education, as well as in the scientific research. In order to take such a geophysical advantage between different hemispheres, we have developed a remote access system of the telescopes between ITB and GAO (Taguchi et al. 2005). It can provide special opportunities for the astronomical experiences of the opposite hemisphere for various people of each country. In June 2006 we had an experiment of the remote system for the public education. It was completely successful with about 100 people gathering at GAO (Fig. 4). While it was rainy at GAO at that time as it was the wet season of Japan, the participants could enjoyed the southern hemisphere of Bosscha observatory with real time communications between us directly made through the remote system. We could realize that such an event is really exciting and impressive for all the participants. In fact, most of them raised their hopes for the next occasion of such a remote observation of the distant world. We are planning another remote observation of the northern hemisphere from Indonesia next December.

\section{Acknowledgements}

We would like to acknowledge the continuous encouragement by Prof. B. Hidayat in our cooperative activities.

\section{References}

Hashimoto, O., Kingugasa, K., Nishihara, E., Taguchi, H., Malasan, H.L., Kurata, T., Okuda, H., Shimizu, M. \& Kozai, Y. 2002, Proc. The 8th IAU Asian-Pacific Regional Meeting, Vol.II, p. 7

Hashimoto, O., Malasan, H.L., Taguchi, H., Kurata, T., Yamamuro, T., Takeyama, N., \& Shimizu, M. 2005, Proc. The 9th IAU Asian-Pacific Regional IAU Meeting 2006, p. 295

Kinugasa, K., Malasan, H.L., Indradjaja, B., Hashimoto, O., Taguchi, H., Kurata, T., Kozai, Y., \& Hidayat, B. 2005, Proc. The 9th IAU Asian-Pacific Regional IAU Meeting 2006, p. 102

Puri Jatomiko, A.T., Malasan, H.L., Hashimoto, O., \& Taguchi, H. 2005, Proc. The 9th IAU Asian-Pacific Regional IAU Meeting 2006, p. 338

Taguchi, H., Kinugasa, K., Indradjaja, B., Prasetyono, G.I., Setyanto,H., Malasan, H.L., Hashimoto, O., \& Kurata, T. 2005, Proc. The 9th IAU Asian-Pacific Regional IAU Meeting 2006, p. 102

Takeda, Y. Hashimoto, O., Taguchi, H., Yoshioka, K., Takada-Hidai, M., Saito, Y., \& Honda, S. 2005, PASJ 57, 751

Wiramihardja, S.D. 2003, Effective Teaching and Learning of Astronomy, 25th meeting of the $I A U$, Special Session 4 